\title{
A Study of Multiscale Density Fluctuation Measurements
}

\author{
Nils P. Basse, Member, IEEE
}

\begin{abstract}
Intriguing parallels between density fluctuation power versus wavenumber on small $(\mathrm{mm})$ and large $(\mathrm{Mpc})$ scales are presented. The comparative study is carried out between fusion plasma measurements and cosmological data. Based on predictions from classical fluid turbulence theory, we argue that our observations are consistent with 2D turbulence. The similar dependencies of density fluctuations on these disparate scales might indicate that primordial turbulence has been expanded to cosmological proportions.
\end{abstract}

Index Terms-Cosmology, density fluctuations, fusion plasmas, turbulence, wavenumber spectra.

\section{INTRODUCTION}

It is a very human trait to compare new observations to previous experience. Our chance encounter with measurements of the spectral power of density fluctuations on Mpc scales lead us to the conclusion that corresponding mm scale measurements in fusion plasmas have surprisingly similar features [1]. We are of the opinion that this correspondence could have a significant impact on current ideas regarding the formation of the universe.

Let us briefly present our reasoning: Fusion plasmas are turbulent, whereas density fluctuations on cosmological scales are not. However, the cosmological fluctuations might be what has been dubbed "fossilized turbulence" [2], [3], i.e. static images of primordial turbulence. This original hot big bang turbulence is in our picture represented by fusion plasma turbulence. So the emerging understanding is as follows: (i) turbulence was generated before the inflationary expansion of the universe, (ii) as the universe cooled and expanded, the primordial turbulence fossilized and is visible on cosmological scales today. The theoretical basis of this hypothesis is outlined in Refs. [4], [5].

We show in this paper that both sets of measurements fit the shape expected from 2D fluid turbulence theory. According to our interpretation, this implies that early turbulence was 2D.

The fusion plasma measurements presented in this paper are of fluctuations in the electron density. Phase-contrast imaging (PCI) [6] is being used in the Alcator C-Mod tokamak [7] and small-angle collective scattering (SACS) [8] was used in the Wendelstein 7-AS (W7-AS) stellarator [9].

We specifically study density fluctuation power $P$ versus wavenumber $k$ (also known as the wavenumber spectrum) in C-Mod and W7-AS. These wavenumber spectra characterize

Manuscript submitted August 23, 2007. This work was supported by the U.S. Department of Energy, Office of Fusion Energy Sciences.

N. P. Basse was with the Plasma Science and Fusion Center, Massachusetts Institute of Technology, Cambridge, MA-02139, USA. He is now with ABB Switzerland Ltd., Corporate Research, Segelhofstrasse 1, CH-5405 BadenDättwil, Switzerland (e-mail: nils.basse@ch.abb.com). the nonlinear interaction between turbulent modes having different length scales. Our explicit assumption is that turbulence in stellarators and tokamaks is comparable.

The second part of our measurements, a cosmological wavenumber spectrum constructed from a variety of sources, has been published in Ref. [10] and was subsequently made available to us [11]. The measurements were used to constrain cosmological variables, e.g. the matter density $\Omega_{m}$ and neutrino masses - for further details see Refs. [10], [12].

The paper is organized as follows: In Sec. I we analyze fusion plasma and cosmological wavenumber spectra. Thereafter we treat the dimensionality of the measurements in Sec. III. We discuss the hot big bang turbulence theory in Sec. IV and conclude in Sec. V.

\section{WAVENUMBER SPECTRA}

We begin by studying the fusion plasma wavenumber spectrum shown in Fig. 11. The plot shows PCI measurements along with a fit to

$$
P\left(k \rho_{s}\right) \propto\left(k \rho_{s}\right)^{-m},
$$

where $\rho_{s}$ is the ion Larmor radius at the electron temperature and $m$ is a constant. The measurements were made in a low confinement mode C-Mod plasma, see Fig. 11 in Ref. [13]. The wavenumbers measured have been multiplied by $\rho_{s}$, which for this case is $0.6 \mathrm{~mm}$. This is the value at $80 \%$ of the plasma radius where the electron temperature is $400 \mathrm{eV}$, the toroidal magnetic field is $6.4 \mathrm{~T}$ and the working gas is Deuterium.

Our fit to the indicated PCI data yields $m=1.0 \pm 0.03$.

All fits shown in this paper have a normalized $\chi^{2} \leq 1$, ensuring a satisfactory quality. The error bars are standard deviations and the semi-transparent rectangles indicate which points are included to make the fits.

In Fig. 2 we show SACS measurements at somewhat larger wavenumbers compared to the PCI data. Again, the measured wavenumbers have been multiplied by $\rho_{s}$, which in this case is $1 \mathrm{~mm}$. This value is also at $80 \%$ of the plasma radius where the electron temperature is $300 \mathrm{eV}$, the toroidal magnetic field is $2.5 \mathrm{~T}$ and the working gas is Hydrogen.

The SACS measurements are fitted to

$$
P\left(k \rho_{s}\right) \propto \frac{\left(k \rho_{s}\right)^{-p}}{1+\left(k \rho_{s} /\left(k \rho_{s}\right)_{0}\right)^{q}},
$$

where $p=2.8 \pm 0.6$ and $q=5.7 \pm 1.3$ are constants. The functional form in (2) is taken from Ref. [15]. Basically this equation describes two power-laws, where $P \propto\left(k \rho_{s}\right)^{-p}=$ $\left(k \rho_{s}\right)^{-2.8}$ for medium wavenumbers and $P \propto\left(k \rho_{s}\right)^{-p-q}=$ 


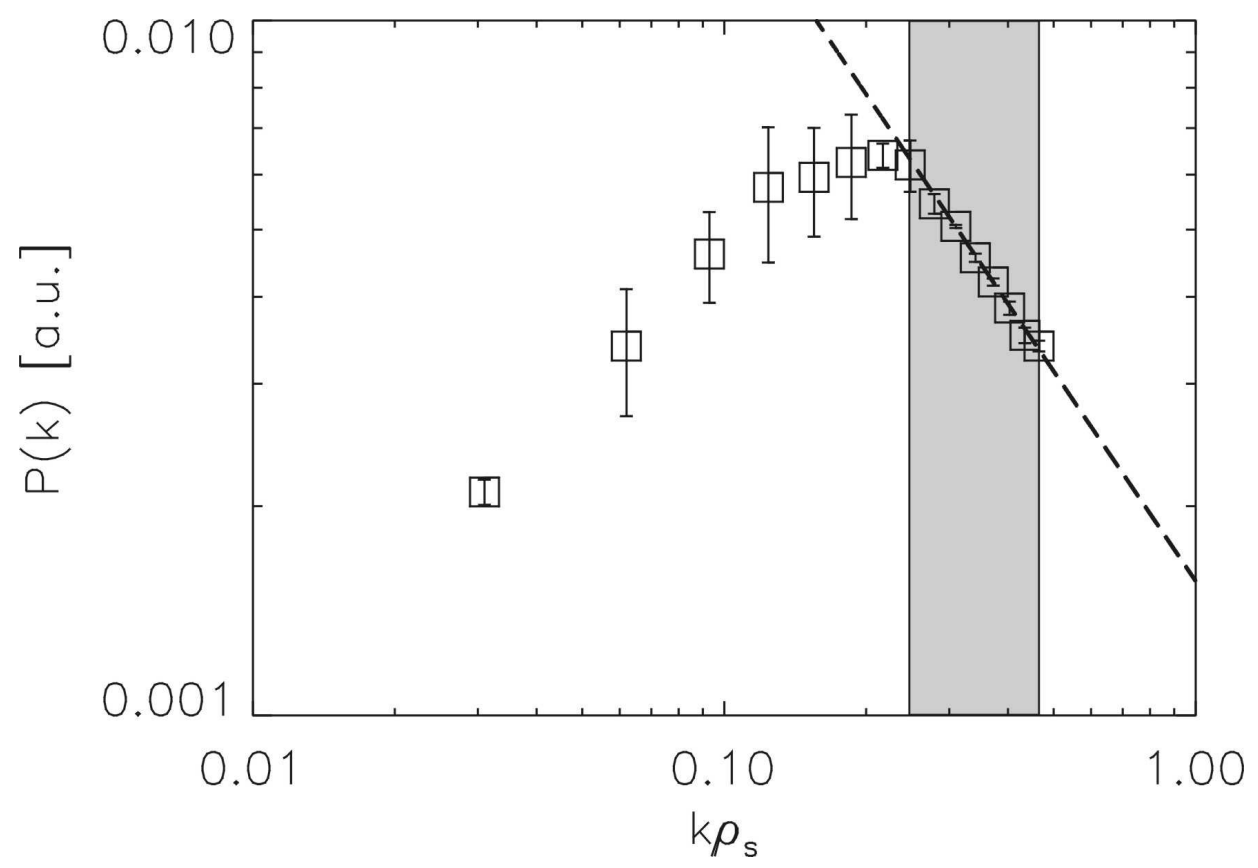

Fig. 1. Wavenumber spectrum of broadband turbulence in C-Mod. Squares are measured points. The dashed line is a fit to 10; the semi-transparent rectangle indicates which points are included to make the fit. The measurements are taken from Fig. 11 in Ref. [13].

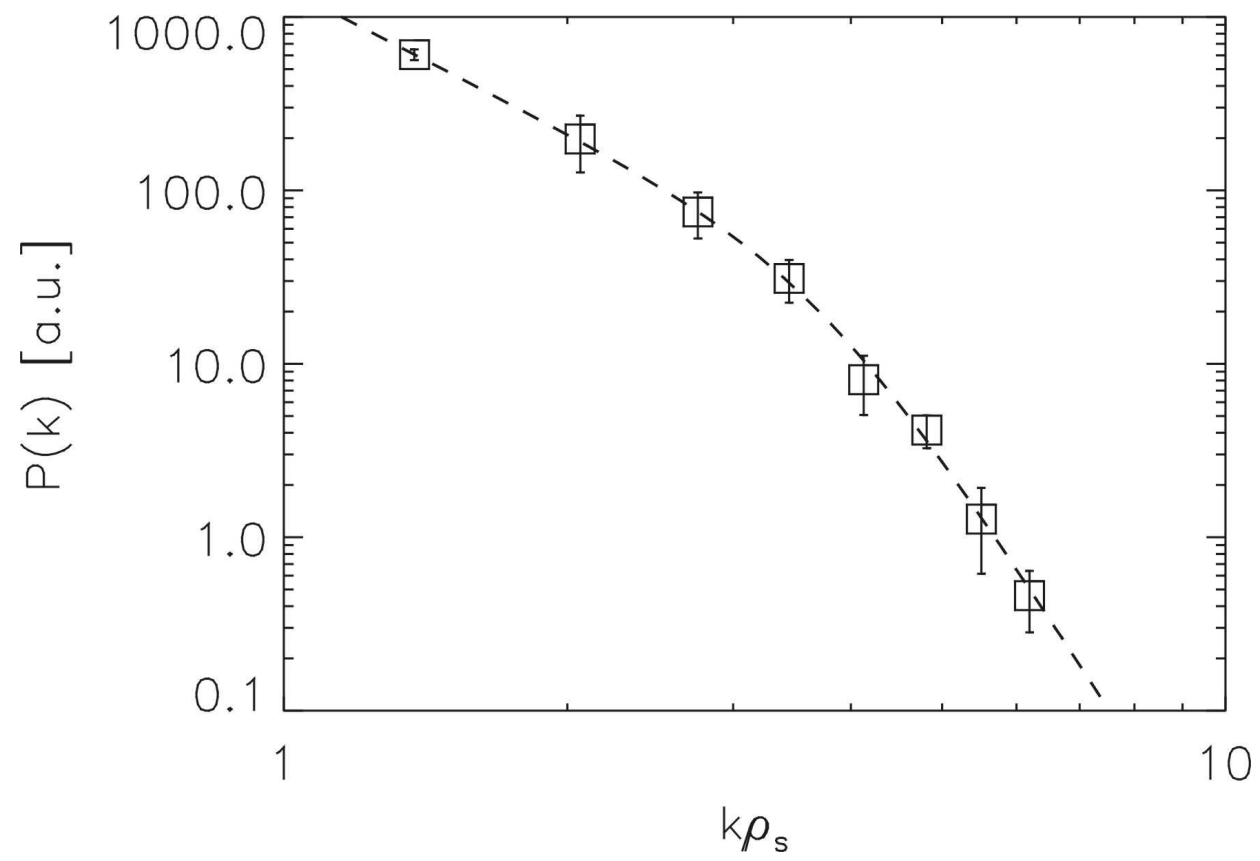

Fig. 2. Wavenumber spectrum of broadband turbulence in W7-AS. Squares are measured points. The dashed line is a fit to 2]; all points are included to make the fit. The measurements are taken from Fig. 12 in Ref. [14].

$\left(k \rho_{s}\right)^{-8.5}$ for large wavenumbers. The transitional $\left(k \rho_{s}\right)_{0}$ is in our case 3.7. The W7-AS data have been taken from Fig. 12 in Ref. [14].

It is at this point relevant to note that the medium wavenumber fusion plasma exponent is not always three (or 2.8), it typically varies between three and four depending on specific plasma conditions [16], [17], [18]. Presumably this is due to different instabilities driving turbulence for varying operating conditions, leading to forcing centered at changing scales.

The cosmological wavenumber spectrum is shown in Fig.
3 The measurements are fitted to (2), but using $k$ instead of $k \rho_{s}$; in this case, $p=1.2 \pm 0.1$ and $q=1.4 \pm 0.05$ are constants. Here, $P \propto k^{-p}=k^{-1.2}$ for small wavenumbers and $P \propto k^{-p-q}=k^{-2.6}$ for medium wavenumbers. The transitional wavenumber $k_{0}$ is $0.3 \mathrm{~h} \mathrm{Mpc}^{-1}$. Here, $h=$ $H_{0} /(100 \mathrm{~km} / \mathrm{s} / \mathrm{Mpc}) \simeq 0.7$, where $H_{0}$ is the Hubble parameter observed today. 


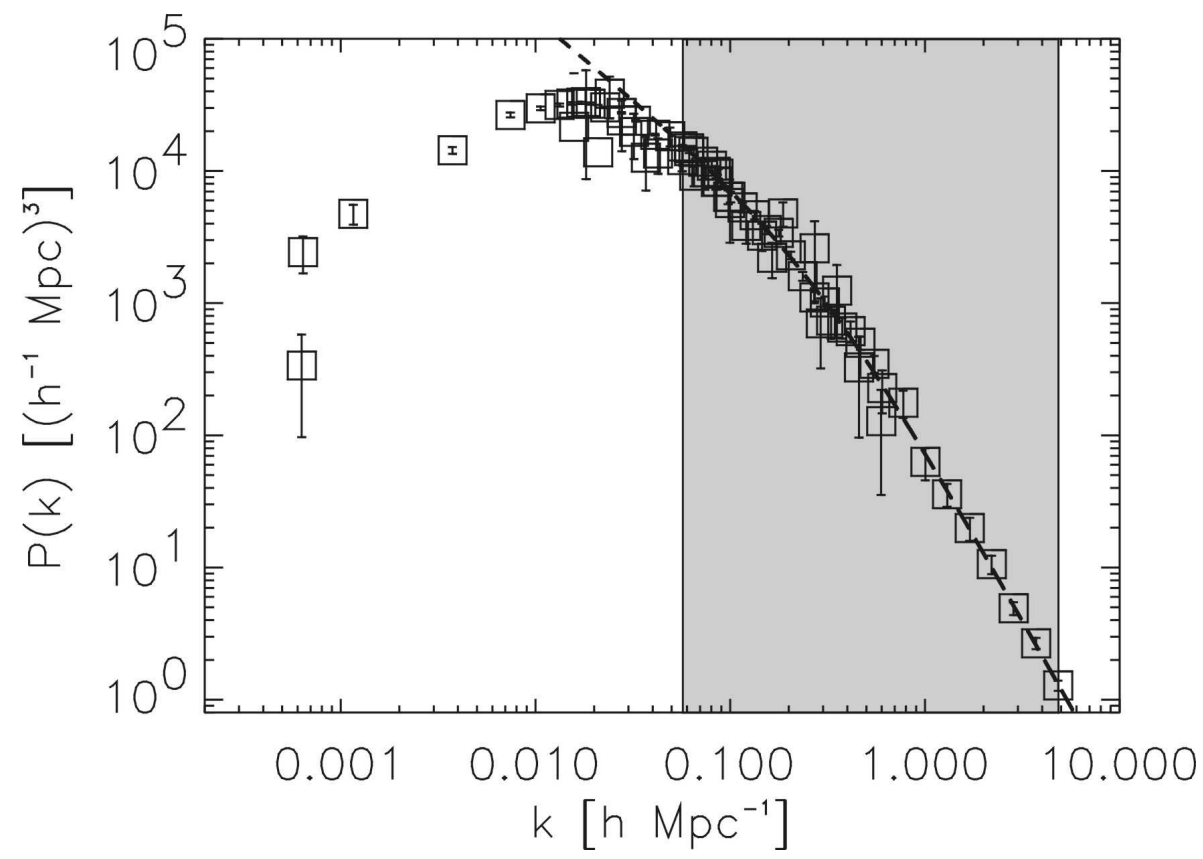

Fig. 3. Wavenumber spectrum of the combined cosmological measurements. Squares are measured points. The dashed line is a fit to 2 ; the semi-transparent rectangle indicates which points are included to make the fit. The measurements are taken from Fig. 38 in Ref. [10].

\section{Dimensionality OF THE MEASURED FLUCTUATIONS}

We begin Sec. III by summarizing our findings on the dependencies of power on wavenumber in Sec. II]

Small wavenumbers : $P(k) \propto k^{-1.0}$ (fusion) or $P(k) \propto k^{-1.2}$ (cosmology).

Medium wavenumbers : $P(k) \propto k^{-2.8}$ (fusion) or $P(k) \propto k^{-2.6}$ (cosmology).

Large wavenumbers :

$$
P(k) \propto k^{-8.5} \text { (fusion). }
$$

Our measured density fluctuation power is equivalent to the $d$-dimensional energy spectrum $F_{d}(k)$ [19], [20], [21]

$$
P(k)=F_{d}(k)=\frac{E(k)}{A_{d}}
$$

$$
A_{1}=2 \quad A_{2}=2 \pi k \quad A_{3}=4 \pi k^{2},
$$

where $A_{d}$ is the surface area of a sphere having radius $k$ and dimension $d$.

We can convert our results in (3) either under the 2D turbulence assumption:

Small wavenumbers : $E(k) \propto k^{0.0}$ (fusion) or $E(k) \propto k^{-0.2}$ (cosmology).

Medium wavenumbers : $E(k) \propto k^{-1.8}$ (fusion) or $E(k) \propto k^{-1.6}$ (cosmology).

Large wavenumbers : $E(k) \propto k^{-7.5}$ (fusion). or under the 3D turbulence assumption:

\section{Small wavenumbers :}

$$
E(k) \propto k^{1.0} \text { (fusion) or } E(k) \propto k^{0.8} \text { (cosmology) }
$$

Medium wavenumbers : $E(k) \propto k^{-0.8}$ (fusion) or $E(k) \propto k^{-0.6}$ (cosmology).

Large wavenumbers :

$$
E(k) \propto k^{-6.5} \text { (fusion). }
$$

The established picture of 2D fluid turbulence is: (i) turbulence is forced on an intermediate scale $k_{\mathrm{f}(2 \mathrm{D})}$, (ii) energy is transferred to larger scales by the inverse energy cascade, $E(k) \propto k^{-5 / 3}$ [22], and enstrophy is transferred to smaller scales by the forward enstrophy cascade, $E(k) \propto k^{-3}$ [23], and (iii) enstrophy is dissipated at the smallest scales [24].

For 3D turbulence the following process occurs: (i) turbulence is forced on a large scale $k_{\mathrm{f}(3 \mathrm{D})}$, (ii) energy is transferred to smaller scales by the forward energy cascade, $E(k) \propto$ $k^{-5 / 3}$, and (iii) energy is dissipated at the smallest scales.

It is interesting to note that in Ref. [25], two dependencies of the 3D energy spectrum on wavenumber in the dissipation range are considered: One is an exponential falloff, the other claims that $E(k) \propto k^{-7}$ and was proposed by W. Heisenberg. This power-law is quite close to the one we found for fusion plasmas at large wavenumbers.

To determine whether $2 \mathrm{D}$ or $3 \mathrm{D}$ turbulence is observed, we consider the power-laws for medium wavenumbers: The exponents should roughly be in the range [-3, -5/3] for $2 \mathrm{D}$ turbulence and about $-5 / 3$ for $3 \mathrm{D}$ turbulence. Equations (5) and (6) indicate that the observed 2D slopes are close to the expected power-laws and that the $3 \mathrm{D}$ slopes are too shallow. We note that the $2 \mathrm{D}$ slopes are closer to the value for the 
inverse energy cascade than for the forward enstrophy cascade. The reason for this is not understood.

Turbulence in fusion plasmas is approximately $2 \mathrm{D}$, since transport along magnetic field lines is nearly instantaneous. For this reason, fluctuations are measured parallel to the major radius of the machine, i.e. perpendicular to the confining magnetic field.

The reason we chose to analyze fusion plasma data was simply a matter of having available measurements and expertise in that field. Any turbulent 2D plasma should display similar characteristics.

\section{HOT BIG BANG TURBULENCE}

In Ref. [1] we suggested that the observed plasma turbulence might originate during an early phase in the formation of the universe. Recent theoretical work on the role of hot big bang turbulence in the primordial universe [5] lends support to this assumption:

In this theory, turbulence observed today was created before cosmological inflation by inertial-vortex forces leading to an inverse big-bang turbulence cascade with a $-5 / 3$ power-law exponent. Turbulence in the plasma epoch has low Reynold's numbers $\sim 10^{2}$ according to this picture and the preceding quark-gluon plasma has a large gluon viscosity that acts to damp the big bang turbulence. The claim is that hot big bang temperature turbulence is fossilized before the universe cools to the Grand Unified Theory strong force freeze-out temperature $10^{28} \mathrm{~K}$. Later, during nucleosynthesis, fossil temperature turbulence was converted to fossil turbulence patterns in e.g. density turbulence [4].

Analysis of power spectra of cosmic microwave background radiation temperature anisotropies shows that they most likely have a turbulent origin, supporting the idea of turbulence generated in the big bang or the plasma epoch [26].

\section{CONCLUSIONS}

The fact that density fluctuations on small (fusion plasma) and large (cosmological) scales can be described by similar functional dependencies, approximately consistent with 2D fluid turbulence, might indicate that (plasma) turbulence at early times has been fossilized and expanded to cosmological proportions.

Our conjecture concerning the primordial turbulence reflected in our wavenumber spectra can be described as follows: Forcing occurs at an intermediate scale $k_{\mathrm{f}(2 \mathrm{D})}$. The inverse energy cascade leads to spectral condensation at large scales and the forward enstrophy cascade leads to enstrophy transfer towards smaller scales. At very small scales, enstrophy is dissipated.

Our interpretation of the measured wavenumber spectra is consistent with the theoretical framework on hot big bang turbulence presented in Ref. [5] and references therein.

\section{ACKNOWLEDGMENT}

This work was supported at MIT by the Department of Energy, Cooperative Grant No. DE-FC02-99ER54512. We thank M. Tegmark for providing the cosmological measurements analyzed in this paper.

\section{REFERENCES}

[1] N.P. Basse, "Density fluctuations on mm and Mpc scales," Phys. Lett. A, vol. 340, p. 456, 2005.

[2] G. Gamow, "On the formation of protogalaxies in the turbulent primordial gas," Proc. Natl. Acad. Sci., vol. 40, p. 480, 1954.

[3] C.H. Gibson, "Fossil turbulence revisited," J. Marine Systems, vol. 21, p. 147, 1999.

[4] C.H. Gibson, "The first turbulence and first fossil turbulence," Flow, Turb. Combust., vol. 72, p. 161, 2004

[5] C.H. Gibson, "The first turbulent combustion," Combust. Sci. Tech., vol. 177, p. 1049, 2005.

[6] A. Mazurenko et al., "Experimental and theoretical study of quasicoherent fluctuations in enhanced $\mathrm{D}_{\alpha}$ plasmas in the Alcator C-Mod tokamak," Phys. Rev. Lett., vol. 89, p. 225004, 2002.

[7] I.H. Hutchinson et al., "First results from Alcator C-Mod," Phys. Plasmas, vol. 1, p. 1511, 1994.

[8] M. Saffman et al., " $\mathrm{CO}_{2}$ laser based two-volume collective scattering instrument for spatially localized turbulence measurements," Rev. Sci. Instrum., vol. 72, p. 2579, 2001.

[9] H. Renner et al., "Initial operation of the Wendelstein 7AS advanced stellarator," Plasma Phys. Control. Fusion, vol. 31, p. 1579, 1989.

[10] M. Tegmark et al., "The three-dimensional power spectrum of galaxies from the Sloan digital sky survey," Astrophys. J., vol. 606, p. 702, 2004.

[11] M. Tegmark, private communication, 2005.

[12] M. Tegmark et al., "Cosmological parameters from SDSS and WMAP," Phys. Rev. D, vol. 69, p. 103501, 2004.

[13] N.P. Basse et al., "Characterization of core and edge turbulence in Land enhanced $\mathrm{D}_{\alpha}$ H-mode Alcator C-Mod plasmas," Phys. Plasmas, vol. 12, p. 052512, 2005.

[14] N.P. Basse et al., "Low- and high-mode separation of short wavelength turbulence in dithering Wendelstein 7-AS plasmas," Phys. Plasmas, vol. 9 , p. 3035, 2002.

[15] T. Padmanabhan and S. Ray, "Power transfer in nonlinear gravitational clustering and asymptotic universality," astro-ph/0511596

[16] C. Honoré et al., "Small scale density fluctuations in Tore Supra: Rupture in the scaling law," Proceedings of the 25th EPS Conference on Controlled Fusion and Plasma Physics, Prague, European Physical Society, PetitLancy, Switzerland, vol. 22C, p. 647, 1998.

[17] S. Zoletnik et al., "Changes in density fluctuations associated with confinement transitions close to a rational edge rotational transform in the W7-AS stellarator," Plasma Phys. Control. Fusion, vol. 44, p. 1581, 2002.

[18] P. Hennequin et al., "Scaling laws of density fluctuations at high-k on Tore Supra," Plasma Phys. Control. Fusion, vol. 46, p. B121, 2004.

[19] H. Tennekes and J.L. Lumley, "A First Course in Turbulence," MIT Press, Cambridge, 1972.

[20] U. Frisch, "Turbulence," Cambridge Univ. Press, Cambridge, UK, 1995.

[21] G. Antar, Ph.D. Thesis, École Polytechnique, 1996.

[22] S. Chen et al., "Physical mechanism of the two-dimensional inverse energy cascade," Phys. Rev. Lett., vol. 96, p. 084502, 2006.

[23] S. Chen et al., "Physical mechanism of the two-dimensional enstrophy cascade," Phys. Rev. Lett., vol. 91, p. 214501, 2003.

[24] S. Chen et al., "Far-dissipation range of turbulence," Phys. Rev. Lett., vol. 70, p. 3051, 1993.

[25] J. von Neumann, "Recent theories of turbulence," in: A.H. Taub (Ed.), Collected Works VI: Theory of Games, Astrophysics, Hydrodynamics and Meteorology, Pergamon Press, Oxford, 1963.

[26] A. Bershadskii and K.R. Sreenivasan, "Extended self-similarity of the small-scale cosmic microwave background anisotropy," Phys. Lett. A, vol. 319 , p. 21,2003

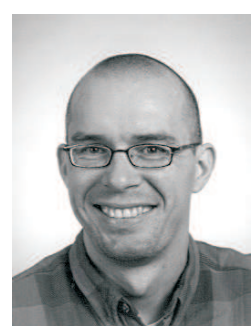

Nils P. Basse received the B.Sc., M.Sc., and Ph.D degrees from the Niels Bohr Institute, University of Copenhagen, Copenhagen, Denmark, in 1996, 1998, and 2002, respectively. He is a Scientist with ABB Switzerland Ltd., Corporate Research. $\mathrm{He}$ was a Postdoctoral Associate at the Plasma Science and Fusion Center, Massachusetts Institute of Technology (MIT), Cambridge, from 2002 to 2005. His present research interests include plasmas in medium- and high-voltage circuit breakers. 